Indian J. Pure Appl. Math., 50(4): 1147-1151, December 2019

(c) Indian National Science Academy

DOI: $10.1007 / \mathrm{s} 13226-019-0381-0$

\title{
ERRATUM TO: V. S. SUNDER, OPERATOR ALGEBRAS IN INDIA IN THE PAST DECADE, Indian J. Pure App. Math., 50(3) (2019), 801-834
}

\author{
Planar algebras in Kolkata in the last decade
}

Due to an unfortunate lack of communication, the input from the Kolkata group of von Neumann algebraists - led by Shamindra Kumar Ghosh and Paramita Das - did not make it in time to be included in the online version of the paper or even in that latest print version of the IJPAM.

\section{SubFACTORS AND $C^{*}$-TENSOR CATEGORIES}

A bifinite bimodule ${ }_{A} \mathcal{H}_{\mathcal{B}}$ over a pair of $I I_{1}$-factors $A$ and $B$ is a Hilbert space $\mathcal{H}$ equipped with commuting actions of $A$ and $B$ from the left and right respectively such that, as one-sided modules, $\mathcal{H}$ is finitely generated. Such bimodules are intimately related to finite index subfactors. For instance, the bimodule ${ }_{A} \mathcal{H}_{\mathcal{B}}$ gives rise to a canonical subfactor $A$ inside $B^{\prime}$ (the commutant of $B$ ). On the other hand, the subfactor $N \subset M$ produces a canonical bimodule ${ }_{N} L^{2}(M)_{M}$ where $L^{2}(M)$ is the GNS Hilbert space of $M$ with respect to the canonical trace. Further, one has a 'dual' bimodule ${ }_{B} \overline{\mathcal{H}}_{A}$ where $\overline{\mathcal{H}}$ is a conjugate Hilbert space of $\mathcal{H}$. Given a pair of such bimodules ${ }_{A} \mathcal{H}_{\mathcal{B}}$ and ${ }_{B} \mathcal{K}_{\mathcal{C}}$, we take

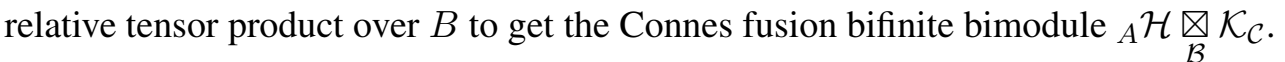

The bifiniteness, duality and Connes fusion gives us a unified view in terms of a semisimple, rigid $C^{*}$-2-category Bim with 0-cells being the $I I_{1}$-factors, 1-cells being the bifinite $A$ - $B$ bimodules and 2-cells being the $A$ - $B$-linear maps. In particular, for a $I I_{1}$-factor $A, \mathbf{B i m}_{A A}$ turns into a $C^{*_{-}}$ tensor category. However, this could often become too large to handle. For instance, if $R$ is the hyperfinite $I I_{1}$-factor, $\boldsymbol{B i m}_{R R}$ accommodates the representation category of any finite group as a full subcategory. It would be much more tractable to fix a bifinite bimodule $X={ }_{A_{+}} \mathcal{H}_{\mathcal{A}_{-}}$and consider the full subcategory $\mathcal{C}^{X}$ generated by multiple Connes fusion of $X$ and $\bar{X}$ alternately and their submodules; so $\mathcal{C}^{X}$ has two 0 -cells denoted by the symbols + and - (corresponding to the $I I_{1}$-factors $A_{+}$and $A_{-}$respectively).

The standard invariant of a finite index subfactor $N \subset M$ of type $I I_{1}$ turns out to be one of the most important invariants which can classify large classes, namely the 'amenable' ones. The information of the standard invariant can also be captured in the categorical data of the 2-category $\mathcal{C}^{X}$ 
where $X={ }_{N} L^{2}(M)_{M}$ (see $\left.[1,27]\right)$. On the other hand, Vaughan Jones axiomatized the standard invariant of a subfactor in terms of algebra of certain pictures on the plane which he called planar algebra. Using graphical calculus of morphisms, a planar algebra was associated to any rigid 2category tensor-generated by a single object and equipped with a pivotal structure in [13]; this was executed on a totally algebraic level. Borrowing the idea of [13], in [9], the authors introduced 'bimodule planar algebra' which corresponds to the 2-category $\mathcal{C}^{X}$ for any bifinite bimodule $X$. They identified the effect of pivotal structure on $\mathcal{C}^{X}$ as spherical invariance in the bimodule planar algebra. The authors also reconstructed a bimodule starting from the bimodule planar algebra without any assumption of sphericality following the reconstruction method prescribed in [21, 22]; however, this was already achieved in the Ph.D thesis of Michael Burns using the technique of Sorin Popa's $\lambda$-lattice (see [24]).

Apart from the above results (which were structural in nature), several examples were worked upon. Given 'outer actions' of a pair of finite groups $H$ and $K$ on a $I I_{1}$-factor $A$, one may consider the fixed point subalgebra $A^{H}$ (of $A$ under the action of $H$ ) sitting inside the crossed product $A \rtimes K$; this family of subfactors was considered by Bisch and Haagerup in [8] and turned out to be extremely useful. Assuming the group generated by $H$ and $K$ in the automorphism group of $A$ has trivial intersection with the inner automorphisms, the associated planar algebra was abstractly characterized in [2], its description bearing close resemblance to IRF models in Statistical Mechanics. If the assumption is dropped, a certain scalar 3-cocycle of the outer automorphism group of $A$ appeared, making the planar algebra quite complicated; however, the description of the bimodule category can be found in [6].

Given automorphisms $\theta_{1}, \cdots, \theta_{n}$ of a $I I_{1}$-factor $A$, the 'diagonal subfactor' is basically $A$ sitting inside $M_{n}(A)$ diagonally but twisted by the automorphisms $\theta_{i}$. The associated planar algebra was characterized in [3] and depended on the subgroup generated by $\theta_{i}$ 's in the outer automorphism group of $A$ and a certain scalar 3-cocycle of it.

Jones noticed that every planar algebra contains a Temperley-Lieb (TL) planar algebra as a canonical sublagebra. So, every planar algebra can be considered as module over a TL planar algebra. The idea of Jones was to attack the existence problem of subfactors given a certain condition on the 'principal graph' or the index by investigating which modules over a TL planar algebra turn out to be a planar algebra by itself. This strategy soon turned out to be extremely useful and helped in construction of several examples such as the 'extended Haagerup subfactor' in [4] and later, in the classification of subfactors with index between 4 and 5 (see [19]).

Another usefulness of these modules is to provide a representation theory for planar algebras. The very first planar algebra was TL whose representation category was obtained in [20]. The next ex- 
ample was the one coming from an outer action of a finite group whose representation was computed in [12] where it was also found that the representation category was additively equivalent to Drinfeld center of the representation category of the group. Analogous results appeared in the world of TQFTs which led Vaughan Jones to reformulate a conjecture of Kevin Walker for the world of subfactors :

Conjecture 1 - If $N \subset M$ is a 'finite depth' subfactor and $P$ is the associated subfactor planar algebra, then the representation category of $P$ is equivalent to the Drinfeld center of the $N$ - $N$-bimodule category $\mathcal{C}_{\mathcal{N N}}$ generated by ${ }_{N} L^{2}(M)_{M}$.

Preliminary results towards settling Conjecture 1 appeared in [10]. Finally, the Conjecture 1 was proved in [11]. Moreover, several nice structures on the representation category of the planar algebra - such as tensor, $W^{*}$, braiding, etc.- were introduced in such a way that the equivalence (in Conjecture 1) preserved these structures. In fact, the authors proved a more general result without the restriction of the finite depth. In the infinite depth case, the representation category is larger than Drinfeld center. Neshvyev and Yamashita (in [26]) found that the representation category is basically the Drinfeld center of a certain induction of $\mathcal{C}_{\mathcal{N N}}$.

The $W^{*}$-structure on the representation category of a planar algebra provides a platform to consider various analytical aspects of it, such as amenability, Haagerup property, property T, etc. This was done in [5] where the authors work in the more general set up of rigid, semisimple $C^{*}$-tensor categories. The idea in the background was to induce the Fell topology on the isomorphism classes of irreducibles and then investigate how the trivial representation can be approximated. It turned out the notions exactly match with those introduced by Popa and Vaes in [25].

There are several examples of planar algebras for which the representation category was studied analytically. The TL case that was already examined by Jones and Reznikoff at an algebraic level, was studied in [5]; the group-type subfactors (Bisch Haagerup and diagonal) in [6]; the case of group twisted categories in [7]. Representation of free product of two arbitrary rigid semisimple $C^{*}$-tensor categories was analyzed in [14]; as an application, the representation of Fuss-Catalan planar algebras was derived.

So far, several results involving planar algebras and $C^{*}$-tensor categories have been encountered. The relation between the two was explored further in [15]. The authors built up a correspondence between rigid semisimple $C^{*}$-tensor categories and 'oriented planar algebras'. When the category is tensor-generated by a single object, the corresponding oriented planar algebra also turned out to be 'singly generated'. Now, every singly generated oriented planar algebra contains a canonical planar algebra associated to some subfactor; there is a forgetful functor in this procedure. Conversely, starting with a subfactor planar algebra, the authors produce a canonical singly generated oriented planar algebra in a functorial way which is 'free' in nature (that is, adjoint to the forgetful functor). 
Using a result of Stefaan Vaes, the authors show that if one starts with a hyperfinite finite index subfactor, the then the free oriented planar algebra corresponds to a $C^{*}$-tensor category which can be embedded in $\boldsymbol{B i m}_{R R}$.

\section{REFERENCES}

1. D. Bisch, Bimodules, higher relative commutants and the fusion algebra associated to a subfactor, The Fields Institute for Research in Mathematical Sciences Communications Series, 13 (1997), 13-63.

2. D. Bisch, P. Das, and S. K. Ghosh, The planar algebra of group-type subfactors, J. Funct. Anal., 257 (2009), 20-46.

3. D. Bisch, P. Das, and S. K. Ghosh, The planar algebra of diagonal subfactors, Quanta of Maths, Proceeding of the Conference in honour of Alain Connes 60th Birthday.

4. S. Bigelow, E. Peters, S. Morison, and N. Snyder, Constructing the extended Haagerup planar algebra, Acta Math., 209(1) (2012), 29-82.

5. S. K. Ghosh and C. Jones, Annular representation theory for rigid $C^{*}$-tensor categories, Jour. Func. Anal., 270(4) (2016), 1537-1584.

6. D. Bisch, P. Das, S. K. Ghosh, and N. Rakshit, Tube algebra of group-type subfactors, Int. Jour. Math., 28(10) (2017), 1750069.

7. J. Bhowmick, S. K. Ghosh, N. Rakshit, and M. Yamashita, Tube representations and twisting of graded categories, Theo. Appl. Cate., 33(31) (2018), 964-987.

8. D. Bisch and U. Haagerup, Composition of subfactors: New examples of infinite depth subfactors, Ann. Sci. Ecole Norm. Sup., 29(1996), 329-383.

9. P. Das, S. K. Ghosh, and V. P. Gupta, Perturbations of planar algebras, Math. Scand., 114(1) (2014), 38-85.

10. P. Das, S. K. Ghosh, and V. P. Gupta, Affine modules and the Drienfeld center, Math. Scand., 118(1) (2016), 119-151.

11. P. Das, S. K. Ghosh, and V. P. Gupta, Drinfeld center of planar algebra, Internat. J. Math., 25(8) (2014).

12. S. K. Ghosh, Representations of group planar algebras, J. Func. Anal., 231 (2006), 47-89.

13. S. K. Ghosh, Planar algebras: A category theoretic point of view, J. Alg., 339(1) (2011), 27-54.

14. S. K. Ghosh, C. Jones, and M. Reddy, Annular representations of free product categories, Jour. Noncomm. Geom., (To appear).

15. S. Ghosh, C. Jones, and M. Reddy, Free oriented extensions of subfactor planar algebras, Int. Jour. Math., 29(13) (2018), 1850093. 
16. V. F. R. Jones, Index for subfactors, Invent. Math., 72 (1983), 1-25.

17. V. F. R. Jones, Planar algebras I, NZ J. Math., arXiv:math/9909027v1 [math.QA].

18. V. F. R. Jones, The annular structure of subfactors, L'Enseignement Math., 38 (2001).

19. V. F. R. Jones, S. Morrison, and N. Snyder, The classification of subfactors of index at most 5, Bull. AMS, 51(2) (2014), 277-327.

20. V. F. R. Jones and S. Reznikoff, Hilbert space representations of the annular Temperley-Lieb algebra, Pac. J. Math., 228(2) (2006), 219-249.

21. V. F. R. Jones, D. Shlyakhtenko, and K. Walker, An orthogonal approach to the graded subfactor of a planar algebra, Pac. J. Math., 246(1) (2010), 187-197.

22. V. Sunder and Vijay Kodiyalam, From subfactor planar algebras to subfactors, Int. J. Math., 20(10) (2009), 1207-1231.

23. A. Ocneanu, Quantized groups, string algebras and Galois theory for algebras, operator algebras and applications, London Math. Soc. Lecture Note Ser., 136 (1988), 119-172.

24. S. Popa, An axiomatization of the lattice of higher relative commutants of a subfactor, Invent. Math., 120(3) (1995), 427-445.

25. S. Popa and S. Vaes, Representation theory for subfactors, $\lambda$-lattices and $C^{*}$-tensor categories, Comm. Math. Phys., 340(3) (2015), 1239-1280.

26. S. Neshveyev and M. Yamashita, Drinfeld center and representation theory for monoidal categories, Comm. Math. Phys., 345(1) (2016), 385-434.

27. V. S. Sunder, $I I_{1}$ factors, their bimodules and hypergroups, Trans. of the Amer. Math. Soc., 330 (1992), 227-256. 